\title{
FEEDBACK ON THE HUMAN FACTORS AWARENESS CAMPAIGN RELATED TO RAILWAY SAFETY AT RATP
}

\author{
SOFIA CHAMI \& FABRICE JUBERT \\ Department of Safety Control, RATP, France
}

\begin{abstract}
Being aware of the propensity for errors is the first step in avoiding making them. This is the goal of awareness efforts undertaken by RATP Co. which, in the very specific case of railway safety, aims to transmit knowledge related to human factors to the Paris metro drivers. The ultimate goal is to have an impact on their behaviors through awareness, in order to continue to strengthen RATP's safety culture. Conceived in a didactic format, the training is made up of 3 major parts. The 1st of which provides a general view on the mechanisms regarding errors, by underlining their diversity. The second describes numerous practices to limit the amount of these errors. The last part focuses on a case study in which we concretely use knowledge mentioned in the preceding sections. Today, this awareness effort is systematically decreasing in the initial trainings offered to commuter rail drivers. It is also being included in the continuing training courses for metro drivers. In order to respond to the increased amount of required training courses, a medium containing videos has been created. A dedicated guide is also available to assist in the transmission of the information. The goal is to provide a training information kit to agents in charge of dispensing this training, in order to increase the spreading of this knowledge, while at the same time maintaining a certain level of coherence in the information communicated.

Keywords: railway safety, risk management, human performance training.
\end{abstract}

\section{INTRODUCTION}

This report is based on the experiment conducted by RATP over a 1 year period training drivers of the Paris Metro on Human Factors.

\subsection{Analysis}

Many railway incidents and accidents are due to human error by drivers [1] or maintainers [2]. In fact, there are very few technical errors which could explain speeding, going through red signals or departing with doors open. They are mainly caused by human error, the primary source of the aforementioned. The Paris Metro does not break rules.

\subsection{Issue}

Based upon this analysis, RATP has decided to make their drivers aware of Human Factors, and more specifically, to make them aware of possible errors when driving. The underlying hypothesis is that the more drivers know about the room for error, the more able they are to avoid them. This is the purpose of promoting such awareness, which in the specific context of railway safety, serves to ensure drivers have a basic understanding of the subject so they can manage their attentional resources as much as possible to specific critical driving moments. The final objective is to use their conscience to define their behaviours, to further reinforce the RATP safety culture.

\subsection{Deployment}

The RATP Metro Department took the ambitious decision to train all drivers over the next two years, by including awareness of Human Factors within their ongoing training. When all is said and done, just under 3,000 drivers will be trained, over 600 training sessions with 
5 drivers per session, in order to avoid affecting line operation too much. In short, this deployment means a great number of training sessions with high expectations from the partaking parties, curious to discover the future advantages of this approach.

\subsection{Presentation}

After having briefly presented the training content, we will focus on the teaching experience and, more specifically, the issues encountered. Then we will present how the design of a new format overseeing awareness enabled us to overcome said issues. To finish off, we will recall the overall view of those who underwent training, as well as their trainers and managers and the result we can expect in terms of railway safety.

\section{TRAINING CONTENT}

Lasting approximately 90 minutes, the training primarily provides an overall view of error mechanisms, focusing on their diversity. Then it provides multiple processes to try and limit their appearance. This is all illustrated using concrete examples taken from different professional sectors. Finally, it focuses on a case study specific to railway where the knowledge tackled in the previous parts is implemented.

\subsection{Theory on error and practices to limit relative emergence}

The first part of training focuses on the main types of human error [3], also looking at the factors which could contribute to the emergence of these completely involuntary mistakes.

In fact, knowledge of mechanisms of human error [4] have presently reached such a point that it is possible to provide a certain amount of information to those who bear the consequences of these mistakes. Drivers are trained on technical malfunctions which they risk facing on their trains and on how to deal with these faults. So why not inform them on their predisposition to error? They are, after all, the main persons concerned. Furthermore, the description of the mechanisms of human error proposed as part of the training is expressed in widely known terms so it is understood by everybody. Additionally, in this job where you continually have to prioritise and often you have to decide which stops (in consideration) to complete to free up resources (reduce mental load) and think about other elements to prioritise, determination of concurrent tasks may turn out to be crucial in terms of railway safety. Defining this determination in light of errors to which a driver is susceptible to commit allows you to give substance, and primarily, sense to the discourse on the importance of prioritising tasks within the driving cabin, particularly the management of communications. What is more, clearly differentiating between the mechanisms of error leads to improved handling of the aforementioned. In essence, the way a careless mistake and a lack of knowledge are handled varies. To select the correct approach, you must know what kind of error you are dealing with.

Beyond simple information, awareness also involves, based upon advances in social sciences - particularly neuroscience - practices enabling better management of attentional resources when it comes to responding to limit errors [5]. These simple to use processes are designed for drivers. Their usefulness is directly checked during the session using exercises completed by drivers during training. The objective is to convince them through practice.

\subsection{Examples}

All the theoretical content is systematically illustrated using concrete examples. The objective is to never leave the driver alone with the theory, but to always guide them 
through to the practical. Initiating dialogue between theory and practical enables the establishment of knowledge in the head of the driver using clear examples.

The examples selected are taken from different professional sectors: the military, hospital, nuclear and aerial. For example, the air disaster of 1989 on the Varig 254 flight from Marabá to Belém in Brazil was due to a succession of pilot errors. The pilots were listening, whilst flying, to the Brazil-Chile match, and did not notice they were heading in the wrong direction (Fig. 1).

Further examples, more specific to the railway field, are also spoken of in more detail, notably the collision of two trains on our operating network at the AUBER station in 1981 following the addition of a new signal (Fig. 1).
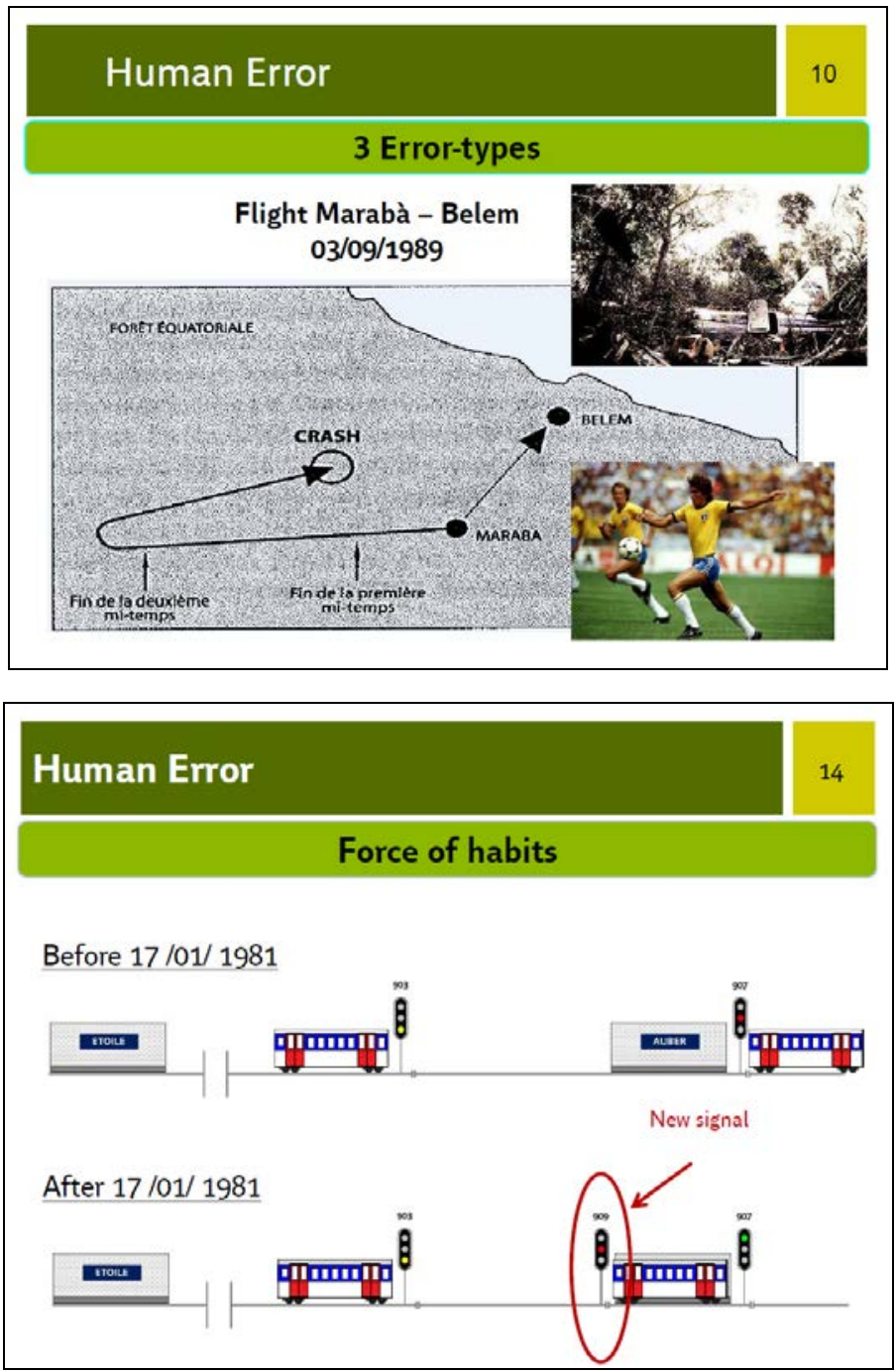

Figure 1: Slides depicting accidents. 


\subsection{Implementation}

The final part of this awareness is dedicated to a practical case from a Human Factor study completed on departures with open doors which have occurred on the Metro network these past years [6]. The idea is to enable drivers to work on incidents so that they concretely use the previously evoked concepts. The hypothesis behind this approach is that you reflect more upon an issue when confronted with the aforementioned, rather than when someone else describes how it has been resolved. Persons undergoing training analyse a whole host of driver post-incident statements to categorise the errors, the causes, to then offer solutions. These solutions are placed in context and discussed in a session.

Convinced of the utility of training for drivers, the RATP Metro Department management wanted all their representatives to benefit from the aforementioned. However, the teaching experience is not without difficulty.

\section{FEEDBACK ON TEACHING}

The challenge was to provide awareness to about 3,000 drivers in pairs, across 600 training sessions, whilst upholding our main network control and surveillance activities.

\subsection{Envisaged measures}

To achieve this, the first measure envisaged was to provide a training session for future organisers selected by the lines, then to guide them through a session before they set off on their task. The future organisers were the direct managers of the drivers. These management representatives are, in fact, in charge of the training of subordinates.

\subsection{Implementation in the terminus}

The first experiment took place on line 11. This measure quickly showed its limitations. Two training sessions with organisers were not adequate. In fact, as they were not experts in social sciences, the organisers did not feel comfortable providing training, even where they were provided with a presentation with slides with commentary. In the end, the messages provided were no longer uniform and sometimes were lacking in consistency, resulting in lots of misunderstandings in the heads of the drivers. This gave the impression that trainers became psychologists in just a few days, leading some drivers to question the legitimacy of their managers to bring up this topic.

This first failed experiment made space for a second which took place on line 3. Using this unfortunate first experience, we wished to reinforce our presence amongst organisers who could better internalise the main messages to disseminate. We supported them over a month providing drivers with training in their presence.

\subsection{Too great a request for a reduced trainer workforce}

In this light, a first awareness session which grouped together line operation representatives (management representatives, terminus management, technical manager, transport manager, etc.) was provided with the aim of presenting the module. This session was followed by 3 support sessions for each management representative, within real situations, during ongoing training. This experiment lasted almost a month, with, in total, about fifteen awareness sessions provided. 
Despite an increase in the number of guided sessions, the management representatives in charge of providing awareness did not manage to adapt to the module. This module is simply a small part of that presented to drivers during ongoing training sessions.

They wanted the first two trainers to provide all awareness training, which was impossible due to the small number of initial trainers (the 2 creators) who could provide training and the great number of awareness elements to implement. Faced with this statement, we had to find an alternative to lighten the tasks of management representatives without burdening initial trainers too much.

\section{DESIGN OF A NEW FORMAT}

In the light of the growing demand from management representatives, we had to rapidly find an alternative solution in order to help them provide the module. The envisaged solution was the creation of a new presentation format.

\subsection{Video supports}

The first envisaged alternative was to film an entire presentation, within a real situation in the presence of drivers, the aim being to ensure the message was provided by the initial trainers, reducing the role of management representatives.

The solution was immediately discarded owing to the inertia of the presentation and the lack of dynamism it provided, which would have provided the reverse effect of that envisaged. The targeted aim was not to completely unburden the management representatives from this task, but rather to help them distribute the module.

An alternative solution therefore had to be found, which would provide the correct messages, whilst keeping some involvement of future trainers in presenting the module. This urged us $t o$ reflect on a presentation format enabling, on the one hand, that main messages are provided by the initial trainers, and on the other hand, that activities can be completed by management representatives.

This lead to the creation of a support in PowerPoint presentation format, which is unique in terms of the integration of small video sequences which introduce different concepts and provide one or two simple and concrete examples to enable immediate understanding (Fig. 2).

The objective was to establish a new support as quickly as possible and to provide it to management representatives. A very short amount of time was given to create this support, so the video sequences were shot by organisers using amateur equipment: A Smartphone! The videos were rapidly created, without the content being neglected.

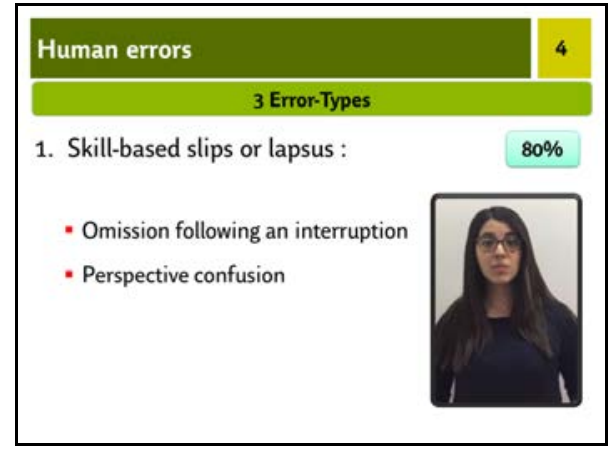

Figure 2: New presentation slides. 
The choice was made to select the most important ideas to introduce, leaving the management representative free to complete the others, providing a certain degree of flexibility and dynamism to the presentation.

Videos were added to the presentation in order to follow the same logic as the initial training, except that, in order to explain each concept, a video is launched.

The support was designed in such a way so it could be immediately assimilated and the videos were launched with a simple click and stopped themselves once finished, providing enough time for the session organiser and trainee drivers to discuss.

\subsection{Activity guide}

An activity guide accompanies this activity presentation. Designed in an educational way, this guide takes the form of a 4-page front/back booklet (Fig. 3).

This guide serves to complete the presentation provided on the USB key. It helps organisers send messages contained in the 26 slides which form the training. Each slide includes comments. The guide also indicates when explanatory videos should be launched.

This activity kit, provided to certified representatives, serves to improve dissemination of Human Factor knowledge, whilst upholding a certain consistency in terms of the information provided.

\section{CONCLUSION: FEEDBACK ON TRAINING}

To conclude, we wish to share the various feedback received on this awareness.

\subsection{Positive feedback from drivers}

We interviewed people who provided the training to find out what the drivers thought
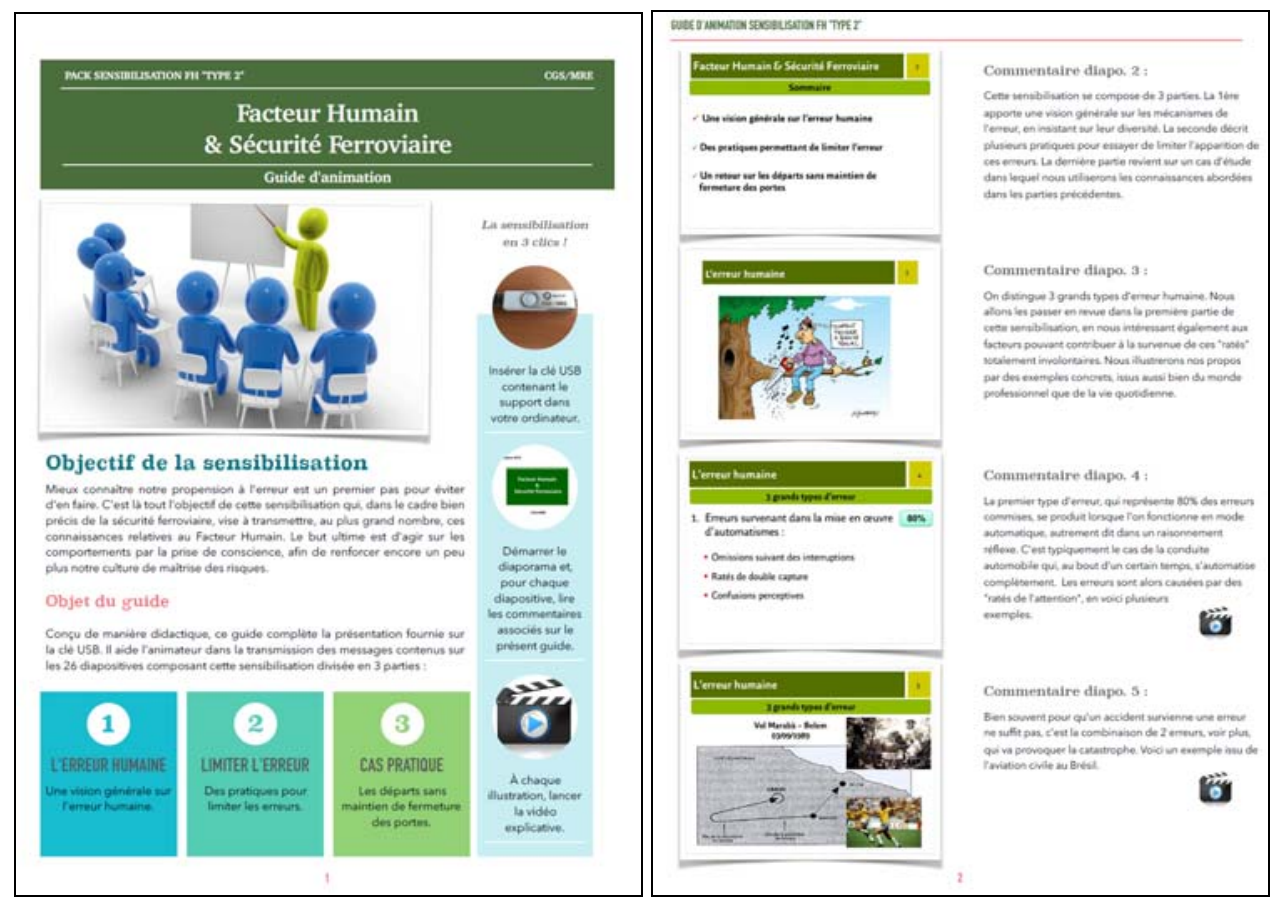

Figure 3: Activity guide. 
of the training. Here are some of the responses received via email:

"The drivers who attended the ongoing training really appreciated it. I had the chance to see the initial trainers once: this was much better. They clearly informed drivers and myself about everything that could disrupt our actions. I found it very useful to use concrete and significant examples (notably, an air plane flight during a football game, as well as examples taken from the work environment of drivers: disruptions during door closure, etc.). Significance was provided, which was good."

"The drivers were captivated; the context scenarios provided sense to the training. Additionally, it is a new section in the ongoing training, which injects freshness into the session."

"In terms of the drivers, the many examples and the small quizzes ensured they were not too bored. In the same way, where the initial trainers could not attend, the drivers found the videos very clear and engaging."

"The drivers are delighted with the awareness of human factors as it focused on correct actions to adopt to avoid errors. They took into consideration the importance of the human factor."

The statement below particularly caught our attention as it testifies the importance of training and, specifically, a feasibility process tackled during awareness:

"The participants appreciated the training, and after a few days, appeared to show willingness to apply the 'verbalisation' to avoid crossover primarily."

The witness accounts gathered directly from drivers confirm the positive feedback of the management representatives. Subsequent to training, the drivers declared being less stressed during driving tasks, but confirm that they apply greater vigilance during situations which may lead to error.

\subsection{Support for organisers}

This perception is shared by organisers. They specifically appreciate the ease of use of this new support and how it can be used to correctly pass on messages. Here is some feedback:

"I find that the training is very well done and highlights the human errors which we could face thanks to clear examples (such as a plane crash). The presentation videos are a precious support to the PowerPoint presentation."

"I then used the PowerPoint with the videos where the initial trainers were not available. It is a good support which successfully replaces them, even though it is nicer in their presence. I found the training essential!"

"The support available is very easy to access and loyally reflects their intervention within the field during activities."

\subsection{Satisfied management}

The Metro Department Management, as well as that of different lines are happy with the training. They find that the training was appreciated by the drivers - at times, drivers state 
that the awareness has resulted in the change of some elements when driving. The ongoing training was deployed on lines 7, 10, 9, 4, 2 using the new presentation format.

\subsection{Awaiting railway safety error results}

It is still too early to assess the effects of awareness on driver behaviour. However, we have noted a reduction in driving discrepancies on certain lines where training has been provided. It is to be continued for the long-term in order to ensure that this awareness has positive results.

\section{REFERENCES}

[1] Bert, J.P. \& Jubert, F., Rolling-stock Change Risk Management: An Innovative Approach. The Sustainable World, ed. C.A. Brebbia, Wessex Institute of Technology: UK, 2010.

[2] Bert, J.P. \& Jubert, F., Managing the risks associated with a basic yet critical maintenance operation in the Paris metro workshops. Rail Human Factors: Supporting reliability, safety and cost reduction, eds Dadashi, N, Scott, A, Wilson, J.R. \& Mills, A., CRC Press, 2013.

[3] Rasmussen, J., Human errors: taxonomy for describing human malfunction in industrial installations. Journal of Occupational Accidents, 4, pp. 311-335, 1982.

[4] Reason, J., Human error, Cambridge University Press: Cambridge, 1990.

[5] Theurier, J.P., Le projet Performance Humaine, Engineering Production Department, Nuclear Production Division, 2010.

[6] Jubert, F., Human-based solutions for open-door train departures, Sustainable Development, ed. C.A. Brebbia, Wessex Institute of Technology: UK, 2015. 